REVISTA DE LITERATURA E CULTURA RUSSA

\title{
Subjectivité de la représentation du genre littéraire chez Jouri Tynianov et Mikhail Bakhtine : essai de définition et étude de textes
}

\section{Subjectivity of the representation of the literary genre in Yurii Tynianov and Mikhail Bakhtin: attempt at definition and study of texts}

Autor: Svetlana Garziano

Edição: RUS Vol. 11. № 16

Data: Setembro 2020

https://doi.org/10.11606/issn.2317-4765.rus.2020.171719 


\section{Subjectivité de la représentation du genre littéraire chez Jouri Tynianov et Mikhaill Bakhtine: essaì de définition et étude de textes}

Résumé: Afın d'approfondir l'examen de la nature du genre littéraire et comme le présent volume porte en partie sur les questions du formalisme russe, cet article s'intéressera à l'expression de la subjectivité appliquée aux genres littéraires telle qu'elle est exposée dans quelques textes fondamentaux écrits par deux grands théoriciens du verbe russe : d'une part, il s'agit de Jouri Tynianov, l'un des principaux acteurs de l'OPOÏAZ, le fondateur du formalisme russe avec Victor Chklovski, Roman Jacobson et Boris Eikhenbaum, et, d'autre part, c'est Mikhaïl Bakhtine qui a entamé son cheminement intellectuel au moment où « le formalisme était au faîte de sa gloire ».

\section{Svetlana Garziano*}

\begin{abstract}
For a deeper examination of the nature of the literary genre and as the present volume is, in part, dedicated to questions of Russian formalism, this article focuses on the expression of subjectivity applied to literary genres as presented in some fundamental texts of two great theoreticians of the Russian word: on the one hand Yurii Tynianov, one of the main figures of the OPOIAZ, founder of Russian formalism along with Viktor Shklovskii, Roman Jakobson and Boris Eikhenbaum; and, on the other hand, Mikhail Bakhtin, who began his intellectual path at the time when «formalism was at the height of its glory».
\end{abstract}

Mots-clés: Genre littéraire; Subjectivité; Jouri Tynianov; Mikhaïl Bakhtine Keywords: Literary genre; Subjectivity; Yurii Tynianov; Mikhail Bakhtin 
* Svetlana Garziano, maître de conférences en langue et littérature russes à la faculté des langues, membre de l'équipe MARGE à la faculté des lettres, directrice de la Maison des langues de l'Université Jean Moulin Lyon 3. Elle a soutenu sa thèse en 2009 et en a publié la version remaniée en 2012 sous le titre de La poétique autobiographique de Vladimir Nabokov dans le contexte de la culture russe et occidentale (Université Jean Moulin Lyon 3, CESAL). svetlana.garziano@univ-lyon3.fr

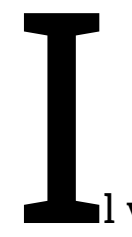

va de soi que la théorie littéraire, depuis La poétique d'Aristote, tend à délimiter les formes littéraires, à les classer et à en montrer l'évolution. La définition du genre littéraire telle qu'elle est exposée dans le Dictionnaire historique de la langue française nous montre justement que la notion de genre littéraire sert à répertorier et à regrouper les œuvres en différentes catégories d'après des critères bien définis. Un genre littéraire, pour obtenir son autonomie, doit en effet acquérir des traits qui nous permettraient de le distinguer des autres:

Genre a d'abord la valeur latine de " catégorie, type, espèce » (v. 1121-1134, gendre), liée au sens de « race » qui a disparu depuis l'époque classique... C'est l'idée générale de « groupement, catégorie » qui domine l'ensemble des emplois au cours de l'histoire. Genre s'emploie par extension, en philosophie (v. 1300) au sens d'« idée générale d'un groupe d'êtres ou d'objets ayant des caractères communs ", puis désigne (1645) une catégorie d'œuvres définie par des caractères communs (sujet, style, etc.)... ${ }^{1}$

Afin d'approfondir l'examen de la nature du genre littéraire et comme le présent volume porte en partie sur les questions du formalisme russe, nous allons recourir dans nos démonstrations à l'expression de la subjectivité appliquée aux genres littéraires telle qu'elle est exposée dans quelques textes fondamentaux écrits par deux grands théoriciens du verbe russe : d'une part, il s'agit de Jouri Tynianov, l'un des principaux acteurs de l'OPOÏAZ, le fondateur du formalisme russe avec Victor Chklovski, Roman Jacobson et Boris Eikhenbaum, et, d'autre part, c'est Mikhail Bakhtine qui a entamé son cheminement intellectuel au moment où « le formalisme était au faîte

1 Le dictionnaire historique de la langue française, Alain Rey (éd.), Paris, Dictionnaires le Robert, 2006, t. 2, p. 1576. 
de sa gloire ${ }^{2}$ Dans son essai sur Mikhaïl Bakhtine de l'Histoire de la littérature russe. Le XXe siècle. Gels et dégels, Michael Holquist précise à juste titre que la position des formalistes sur le texte comme l'ensemble des procédés employés lui a permis de concevoir sa propre théorie littéraire, en s'opposant à deux principaux postulats des premiers formalistes : l'œuvre conçue comme quelque chose de fait, fabriqué et l'omission du critère des conditions historiques. Michael Holquist poursuit sur les traits distinctifs et communs de ces chercheurs pionniers en théorie littéraire : «Si donc les formalistes se concentraient sur les mécanismes de la versification et de la narration $\langle\ldots .>$, Bakhtine, lui, attirait l'attention sur la sémantique de l'œuvre. En cela, il annonçait une tendance qui se manifesta au grand jour dans le formalisme tardif, particulièrement dans l'œuvre de Tynianov. Mais, chez Bakhtine, l'accent est mis sur les facteurs sociaux comme terrain de la signification : l'interprétation apparaît comme une fonction de facteurs venus de l'ensemble de la culture. Ce qui est commun à Bakhtine et aux formalistes (et par la suite aux structuralistes), c'est d'avoir concentré leurs efforts sur le langage... $~^{3}$ Cette concentration sur le langage sera notre fil conducteur tout le long de notre exposé où nous procéderons à la lecture attentive de leurs différents textes théoriques, tout en démêlant des structures permettant des modalités d'une compréhension subjective dans leurs approches respectives.

\section{"De l'évolution littéraire " de Tynianov: évolution à l'oeuvre}

Soit dit en passant, l'idée d'évolution est propre à l'époque de l'Âge d'argent et influencée, en grande partie, par les multiples théories de l'évolution qui surgissent au fil du XIXème siècle. La réflexion sur la langue comme organisme vivant chez les

2 Michael Holquist, « Mikhail Bakhtine », in Histoire de la littérature russe. Le XXe siècle. Gels et dégels, Paris, Fayard, 1990, p. 566.

3 Ibid., p. 567. 
formalistes russes est amorcée par exemple par Victor Chklovski dans son texte très connu "La résurrection du verbe " ["Воскрешение слова», ${ }^{4}$ 1914] et poursuivie par presque tous les formalistes russes.

Dans son essai sur Jouri Tynianov de l'Histoire de la littérature russe. Le XXe siècle. La Révolution et les années vingt, Ilya Serman note que "sa conception de la littérature comme processus de modification permanente de l'ensemble des procédés artistiques - de la construction du sujet à la structure rythmique - Tynianov l'a étendue dans son livre le Problème de la langue du vers (Проблема стихотворного языка, 1923) à « l'analyse des changements spécifiques de la signification et du sens du mot en fonction de la construction même du vers" $» .^{5}$

En effet, "De l'évolution littéraire » «О литературной эволюции», 1927] propose deux critères pour expliquer les processus littéraires : celui de la forme et celui de la fonction. Examinons de près quelle définition-système expose Tynianov dans ce texte. Justement il considère ici le genre littéraire en tant qu'une structure systémique où la fonction joue le rôle de « rivet » qui lie différents éléments formels entre eux. D'où, d'ailleurs, l'utilisation massive de thèmes, de motifs, d'ornements dans l'écriture du XXème siècle comme une réflexion possible sur la combinaison des formes et des fonctions. Le genre littéraire surgit au moment où la forme correspond à la fonction, souligne Tynianov : « Соотнесенность каждого элемента литературного произведения как системы с другими, и, стало быть, со всей системой я называю конструктивной функцией данного элемента »" (« Je nomme fonction constructive d'un élément donné la corrélation de chaque élément d'une œuvre littéraire en tant que système avec les autres éléments et, par conséquent, avec

4 Notons en passant que le terme russe "slovo » veut dire, selon le contexte, verbe, parole, discours, vocable, mot.

5 llya Serman, « louri Tynianov », in Histoire de la littérature russe. Le XXe siècle. La Révolution et les années vingt, Paris, Fayard, 1988, p. 679.

6 Ju. N. Tynjanov, « 0 literaturnoj èvoljucii », Literaturnaja èvoljucija. Izbrannye trudy, Moskva, Agraf, 2002, p. 192. 
le système en entier "). Ce faisant le théoricien propose une approche systémique (selon le modèle de Saussure) qui montre par conséquent le rôle d'un genre littéraire dans un système artistique donné : "Sur ce fondement, nous concluons : l'étude des genres isolés en dehors des signes du système de genre avec lequel ils entrent en corrélation est impossible. Le roman historique de Léon Tolstoï n'est pas en corrélation avec le roman historique de Mikhaïl Zagoskine, mais est en corrélation avec la prose qui lui est contemporaine.. ${ }^{7}$

Or, selon Tynianov, les éléments formels, en nombre toujours limité, évoluent plus rapidement que les fonctions d'un genre littéraire et en acquièrent constamment de nouvelles fonctions : « Функция прозы к стиху остается, но формальные элементы, ее выполняющие, - другие " (« La fonction de la prose par rapport au vers reste, mais les éléments formels qui l'accomplissent sont autres "). De cette façon, la structure du système littéraire peut être comparée à celle du système solaire ou d'un atome : les éléments formels suivent un mouvement centrifuge ; les éléments les plus proches du noyau sont les plus importants et significatifs, représentant les traits caractéristiques du système. Pourtant il se produit un réarrangement constant des éléments formels : des éléments périphériques, comparés aux électrons, sont attirés par le noyau sémantique du genre. L'accumulation des éléments formels dans le noyau se résume en son épuisement à cause de la densité de la production littéraire. Le nombre de fonctions étant limité pour un genre, ce dernier n'arrive plus à contenir le nombre croissant d'éléments formels. Il en résulte une explosion des « rivets » de la structure. Par la suite, ces éléments formels éjectés s'attacheront à d'autres structures littéraires. Nous pouvons justement lire à ce sujet chez Tynianov:

Si nous convenons que l'évolution est une modification de la corrélation des membres du système, c'est-à-dire une mo-

7 Ibid., p. 197.

«На этом основании заключаем: изучение изолированных жанров вне знаков той жанровой системы, с которой они соотносятся, невозможно. Исторический роман Толстого не соотнесен с историческим романом Загоскина, а соотносится с современной ему прозой.»

8 Ibid., p. 197. 
dification des fonctions et des éléments formels, l'évolution s'avère être un " changement » de systèmes. Ces changements portent un caractère plus lent ou plus saccadé d'époque en époque et ne supposent pas de renouvellement ni de remplacement soudains et entiers des éléments formels, mais ils supposent une nouvelle fonction de ces éléments formels. ${ }^{9}$

De cette brève analyse textuelle nous pouvons constater que Tynianov favorise, en 1927, l'étude synchronique des genres littéraires et leur positionnement par rapport à d'autres genres, en écartant leur étude diachronique. Mais n'oublions pas que la notion de système émanant de l'approche synchronique et spatiale pourra entrer en collision avec la notion d'évolution inévitablement fondée sur l'approche diachronique et temporelle. Dans cet essai, Tynianov n'a pas pu ou n'a pas voulu concilier cette pensée contradictoire.

Son attitude négative envers l'approche diachronique peut être expliquée non seulement par le fait qu'elle était l'approche dominante de l'époque, mais aussi par ce que la diachronie échappe à toute idée de système. On peut parler d'un « système » synchronique (en synchronie), mais non d'un système diachronique (en diachronie). Autrement dit, la succession des différents états d'un système dans le temps ne peut que difficilement s'organiser en un système cohérent. Il convient qu'il n'y ait pas de système des transformations, dans le sens où il serait possible de systématiser en quelque sorte le passé et qu'il ne soit pas possible d'appliquer les résultats ou modèles obtenus, le système des transformations, au futur, ce qui signifierait qu'il serait envisageable de prédire à la lettre l'évolution postérieure.

9 Ibid., p. 204.

«Если мы условимся в том, что эволюция есть изменение соотношения членов

системы, т. е. изменение функций и формальных элементов, - эволюция оказывается "сменой" систем. Смены эти носят от эпохи к эпохе то более медленный, то скачковой характер и не предполагают внезапного и полного обновления и замены формальных элементов, но они предполагают новую функцию этих формальных элементов.» 


\section{Unité et continuité du genre littéraire selon Mikhaill Bakhtine}

Par rapport à la pensée originale de Tynianov, Mikhaïl Bakhtine ne propose pas de théories novatrices de l'évolution du genre littéraire, mais approfondit l'étude de sa structure et de ses missions. Il considère qu'à des époques diverses, le noyau sémantique d'une langue vivante et son devenir s'assument et s'expriment dans différents genres. ${ }^{10}$ Son étude « Le problème des genres langagiers » [«Проблема речевых жанров»] extraite du livre L'esthétique de la création verbale [Эстетика словесного творчества, 1986] en dit long à ce sujet, en examinant justement la problématique sémantique des genres. Pour Bakhtine, le genre a une importance toute particulière, et il peut être littéraire ou langagier. Il est caractérisé par la présence de trois composantes : la structure, le style et le thème qui déterminent sa spécificité:

Tous ces trois moments, écrit-il, le contenu thématique, le style et la construction de la composition, sont indissolublement liés dans l'énoncé intégral et sont pareillement définis par la spécificité de la sphère de communication donnée. Chaque énoncé isolé est, bien sûr, individuel, mais chaque sphère d'utilisation de la langue produit ses types relativement constants d'énoncés que nous appelons genres langagiers. ${ }^{11}$

Dans « La réponse à la question de la rédaction du Novyï mir » [“Ответ на вопрос редакции “Нового мира”», 1986], Bakhtine poursuit sa réflexion sur la nature des genres. D’après lui,

10 M. M. Baxtin, « Problema teksta », Avtor i geroj. K filosofskim osnovam gumanitarnyx nauk, Sankt-Peterburg, Azbuka, « Academia », 2000, p. 316.

11 M. M. Baxtin, « Problema rečevyx žanrov », Ėstetika slovesnogo tvorčestva, Moskva, Iskusstvo, 1986, p. 250.

«Все эти три момента - тематическое содержание, стиль и композиционное построение - неразрывно связаны в целом высказывании и одинаково определяются спецификой данной сферы общения. Каждое отдельное высказывание, конечно, индивидуально, но каждая сфера использования языка вырабатывает свои относительно устойчивые типы таких высказываний, которые мы и называем речевыми жанрами.» 
dans le genre se cristallisent justement toutes les formes de la perception de l'univers. Dans leur manière d'utiliser le genre littéraire l'opposition entre écrivain-artisan et grand artiste fait ressortir sa double tâche : donner et conserver à la fois les matrices artistiques ainsi que renouveler l'expression esthétique en assurant le lien entre tradition et modernité. Bakhtine écrit:

Les genres jouent un rôle particulièrement important. Dans les genres (littéraires et langagiers), les formes de vision et de compréhension de certains aspects du monde s'accumulent durant les siècles de leur vie. Pour un écrivain-artisan, le genre sert de patron externe, un grand artiste éveille les possibilités de sens qu'il contient. ${ }^{12}$

La définition du genre littéraire est magistralement exposée sous l'angle des éléments archaïsants et novateurs dans Les problèmes de la poétique de Dostoïevski [Проблемы поэтики Достоевского, 1929]. Pour Bakhtine, le genre littéraire de par sa nature même reflète les tendances les plus constantes de l'évolution littéraire : ses éléments archaïques y sont sauvegardés grâce à son renouvellement constant, à son adaptation à la modernité ; le genre est toujours ambivalent, à la fois nouveau et ancien, il se renouvelle à toute étape de l'évolution littéraire par le biais des œuvres autonomes. Le caractère archaïque préservé dans le genre est capable de se renaître sans cesse : « Le genre vit du présent, mais il se rappelle toujours son passé, son origine. Le genre est le représentant de la mémoire créatrice dans le processus du développement littéraire. C'est justement pour cela que le genre est capable d'assurer l'unité et la continuité de ce développement. $»^{13}$ Bakhtine con-

\footnotetext{
12 M. M. Baxtin, "Otvet na vopros redakcii "Novogo mira" ", Èstetika slovesnogo tvorčestva, op. cit., p. 351

«Особо важное значение имеют жанры. В жанрах (литературных и речевых) на протяжении веков их жизни накопляются формы видения и осмысления определенных сторон мира. Для писателя-ремесленника жанр служит внешним шаблоном, большой же художник пробуждает заложенные в нем смысловые возможности.»

13 Mixail Baxtin, Problemy poètiki Dostoevskogo, Moskva, Sovetskij pisatel', 1963, p. 141142.

«Жанр живет настоящим, но всегда помнит свое прошлое, свое начало. Жанр представитель творческой памяти в процессе литературного развития. Именно поэтому жанр и способен обеспечить единство и непрерывность этого развития.»
} 
sidère donc que le genre assure un développement permanent des formes littéraires, la continuité et l'unité de ces différents aspects. Les éléments archaïques qui se renouvellent constamment assurent la vitalité du genre littéraire qui est toujours basé sur la tradition, c'est-à-dire sur la mémoire vivante des siècles passés. Dans cette définition, en considérant le genre littéraire comme un organisme vivant par le biais de multiples personnifications, le théoricien applique le modèle interprétatif biologique à l'évolution des genres.

\section{Émergence de la subjectivité dans les genres littéraires chez M. Bakhtine}

Dans le recueil d'études Questions de littérature et d'esthétique [Вопросы литературы и эстетики, 1975] écrit en 19371938, Mikhaïl Bakhtine examine les formes du temps et du chronotope dans le roman européen. Cet ouvrage nous servira de fil conducteur dans l'analyse de l'émergence de la subjectivité du discours dans l'Antiquité ${ }^{14}$ Le chercheur commence son étude par le roman grec dans lequel il distingue trois types: le roman d'aventures et d'épreuves, le roman d'aventures et de mœurs et le roman biographique. L'antiquité avait créé certaines formes biographiques qui ont exercé une influence profonde sur le développement des genres autobiographique et biographique ainsi que du roman en Europe occidentale. Ces formes reflètent tout particulièrement la prise de conscience publique de l'individu. Bakhtine distingue notamment le temps biographique et l'image dynamique de l'homme : « В основе этих античных форм лежит новый тип биографического времени и новый специфически построенный образ

\footnotetext{
14 Notons à ce propos que la classification du système verbal des langues européennes, qui commence toujours par la première personne du singulier, est héritée de la grammaire grecque. De cette manière purement grammaticale, la Grèce antique a mis, en quelque sorte, en avant la subjectivité propre à l'expression de la première personne du singulier dont les civilisations européennes héritent par la suite. Le cas inverse peut être illustré par la classification des formes verbales en Inde, où la grammaire hindoue cite en premier lieu les verbes à la troisième personne. La prépondérance de la première personne grammaticale du singulier dans les civilisations européennes témoigne, entre autres, de l'attention toute particulière qui est portée au «je », à la personnalité, à l'expression de la subjectivité.
} 
человека, проходящего свой жизненный путь »" (" $\grave{A}$ la base de ces formes antiques se trouve un nouveau type de temps biographique et une nouvelle image spécifiquement construite de 1 homme qui poursuit son chemin de vie ").

Le chercheur suppose que la Grèce classique a connu deux types d'écriture subjective : le type platonicien et le type rhétorique. Le premier type s'est exprimé dans L'apologie de Socrate et Le Phédon de Platon. Pour ce premier type, le temps biographique du héros métamorphosé se dissout dans le temps idéal. Le type platonicien est caractérisé par les formes de métamorphose mythologique, où le chronotope est construit autour de la recherche de la véritable connaissance:

Ce type de connaissance de soi de l'homme est lié aux formes strictes de la métamorphose mythologique. À sa base se trouve le chronotope : « le chemin de vie de celui qui cherche la vraie connaissance ». La vie de cet homme qui cherche se divise en époques ou étapes parfaitement délimitées. Le chemin, passant par une ignorance présomptueuse, un scepticisme autocritique et la connaissance de soi, conduit à la vraie connaissance (mathématique et musique). ${ }^{16}$

La recherche platonicienne de la connaissance s'accompagne du passage par des écoles philosophiques à l'époque hellénistique et romaine:

Ce premier schéma platonicien du chemin de l'homme qui cherche se complique d'éléments extrêmement importants sur le sol grec et romain : le passage de l'homme qui cherche par une série d'écoles philosophiques dans le but d'en éprouver la validité et l'orientation de la division temporelle du chemin sur ses propres œuvres. ${ }^{17}$

\footnotetext{
15 Mixail Baxtin, « Formy vremeni i xronotopa v romane. Očerki po istoričeskoj poètike», op. cit., p. 81

16 Ibid., p. 281.

«Этот тип автобиографического самосознания человека связан со строгими формами мифологической метаморфозы. В основе ее лежит хронотоп - "жизненный путь ищущего истинного познания". Жизнь такого ищущего расчленяется на точно отграниченные эпохи или ступени. Путь проходит через самоуверенное невежество, через самокритический скепсис и через познание самого себя к истинному познанию (математика и музыка).»

17 Ibid., p. 281.

«Эта ранняя платоновская схема пути ищущего на эллинистически-римской почве осложняется чрезвычайно важными моментами: прохождение ищущего через ряд
} 
Le second type, type rhétorique, est issu de l'enkomion, éloge funèbre et commémoratif du citoyen. Il peut être illustré par le plaidoyer d'Isocrate (Bakhtine ne précise pas de quel plaidoyer il s'agit en particulier). Le chronotope intérieur (le temps et l'espace de la vie décrite) fait place au chronotope extérieur et réel, représenté par la place publique, l'agora, qui joue un rôle primordial dans l'évocation de la vie personnelle ou de la vie d'autrui. Sur cette place commence à se former la prise de conscience de l'individu. Par conséquent, le type rhétorique est un acte verbal qui glorifie et justifie en soi l'homme empirique.

À cette période, il n'existe pas de différences génériques entre l'écriture autobiographique et la biographie, car l'homme est entièrement public et extérieur. À l'époque hellénistique et romaine, la question du bien-fondé de l'autoglorification est soulevée par Tacite, Plutarque et Aristide (ici même Bakhtine ne donne pas non plus d'exemples concrets d'œuvres). Cette polémique constitue justement le point de départ dans la distinction de la démarche biographique et autobiographique par rapport à l'existence humaine, dans le traitement différent de la vie de soi et de la vie d'autrui:

Voilà pourquoi derrière la question particulière de la possibilité de l'autoglorification se cache une question plus générale, celle de la possibilité d'appliquer la même approche à sa propre vie et à celle d'autrui, à soi-même et à autrui. La question ainsi posée révèle le fait que $l$ intégrité publique classique de l'homme se désagrégeait et que débutait la différenciation de principe des formes biographiques et autobiographiques. ${ }^{18}$

Ainsi commence le transfert des sphères de l'existence humaine "vers un registre muet et vers une invisibilité de principe » («на немой регистр и на принципиальную

философских школ с испытанием их и ориентация временно́го расчленения пути на собственных произведениях.»

18 Ibid., p. 283.

«Поэтому за специальным вопросом о допустимости самопрославления таится более общий вопрос - о допустимости одного и того же подхода к своей собственной и чужой жизни, к себе самому и к другому. Постановка подобного вопроса говорит о том, что классическая публичная целостность человека распадалась и начиналась принципиальная дифференциация биографических и автобиографических форм.» 
незримость»). ${ }^{19}$ L'image de l'homme, cohérente et extravertie, devient graduellement multiple et introvertie, ses sphères intérieure et extérieure se scindent.

Les écrits subjectifs romains se trouvent dans un autre chronotope réel : c'est la famille romaine qui conserve un caractère public et acquiert un caractère historique et national. Si la conscience autobiographique des Romains est historique et pénétrée par le temps, celle des Grecs est contemporaine et harmonieuse.

Les prodigia, les présages et leur interprétation, sont un trait distinctif de la biographie romaine, un principe structurant de la conception et de l'élaboration du matériau autobiographique naissant et biographique. Aux prodigia se rattache la catégorie du «bonheur " propre aux Romains, bonheur créateur, public et national, qui forme une personnalité et une existence humaine et qui englobe les destins personnels et nationaux. Au cours de l'histoire, la notion de bonheur acquérra un caractère privé et personnel.

À Rome, il subsiste aussi des schémas subjectifs grecques. Les déplorations grecques (naenia) se transforment en panégyriques (laudes). Une autre forme romaine contaminée d'une forme hellénique est le livre sur « les écrits de soi » («o собственных писаниях»), ${ }^{20}$ qui représente un catalogue d'œuvres personnelles, qui traite leur thème, leur succès et qui est accompagné de commentaires autobiographiques (cf. Cicéron). Cette forme est issue du schéma platonicien.

Le théoricien distingue deux types de structures dans la biographie antique, types fondés, comme il le dit lui-même, sur l'essence immuable de l'homme accompli. Le premier type est nommé énergétique, le second type est analytique. Le premier type, dont le représentant éminent est Plutarque, découle du concept aristotélicien d'énergie, il doit décrire non pas un état mais une action, des manifestations et des expressions de l'homme. Son temps biographique est celui de la révélation du caractère qui consiste en l'achèvement de la forme donnée

19 Ibid., p. 285.

20 Ibid., p. 289-290 
à l'homme dès sa naissance. Tandis que le type analytique, représenté par Suétone qui a influencé le genre biographique au Moyen Âge, est basé sur les thèmes et destiné à montrer l'unité d'un caractère. Ces deux types dressent les contours précis de l'unité d'un caractère au début du récit. Le contenu est structuré dans un ordre temporel pour le premier type et dans un ordre systématique pour le second.

Les formes transitoires sont également répertoriées par Bakhtine, elles marquent notamment le passage de la désagrégation de la conscience publique vers le surgissement de la conscience privée et solitaire, passage qui ne fait que s'amorcer dans l'Antiquité. Le théoricien note ainsi trois modifications de cet ordre:

- la représentation satirique et ironique ou humoristique de sa propre vie dans des satires et des diatribes (Horace, Ovide, Properce) ;

- la forme rhétorique intime de l'épître aux amis (lettres de Cicéron à Atticus), où le moi se déplace vers des espaces fermés et intimes, en perdant son caractère public et extraverti, sa plasticité ;

- la modification stoïcienne des consolations (La Consolatio de Cicéron, Les pensées de Marc Aurèle, Sénèque, Les confessions de saint Augustin, Boèce, Pétrarque). Cette modification introduit une nouvelle forme de relation à soi seul sans témoin qui trouve son expression dans le mot « Soliloquia » attribué à saint Augustin : entretiens solitaires avec soi-même. ${ }^{21}$

La description des événements de la vie intime et personnelle s'accroît progressivement dans les écrits biographiques. D'après Bakhtine, le type d'écriture proprement dite subjective commence à se former dans l'Antiquité tardive. Sa genèse est liée à l'intérêt porté à l'individu et à l'essor du genre biographique.

Remarquons que Mikhaïl Bakhtine ne lie pas ostensiblement l'apparition de l'écriture autobiographique au christianisme, cependant il cite à plusieurs reprises l'exemple remarquable de l'émergence d'une conscience "subjective » chrétienne

21 Saint Ambroise de Milan [IVème siècle], le maître de saint Augustin, était, selon le témoignage de ce dernier, la première personne à pratiquer la lecture silencieuse dans l'Antiquité. 
qu'est le livre Les confessions [rédigées entre 397 et 401] de saint Augustin. Cet ouvrage représente le premier exemple se rapprochant de la conception autobiographique d'une œuvre littéraire et inaugurant la tradition des autobiographies spirituelles.

L'homme solitaire n'apparaît qu'au Moyen Âge. C'est sur cette considération que se termine la conclusion de l'étude de Bakhtine.

En conclusion, nous pouvons remarquer que les idées de Jouri Tynianov et de Mikhaïl Bakhtine sur les genres littéraires, malgré leurs diversités, restent toujours pertinentes, productives et valables pour interpréter les faits littéraires. À titre d'exemple, nous pouvons citer l'ouvrage Actes autobiographiques : la situation changeante $d$ un genre littéraire [Autobiographical acts : the changing situation of literary genre, 1976 ${ }^{22}$ où Elisabeth W. Bruss recourt justement à la théorie de l'évolution littéraire de Tynianov. La chercheuse insiste sur le phénomène de la variabilité, en énumérant les variantes qui affectent un genre littéraire. En effet, la notion de variabilité qui fait ressortir la différenciation d'un genre dans le temps aboutit progressivement à l'impossibilité de sa définition. Certes, les genres littéraires évoluent, mais nous pouvons rétorquer aussi qu'ils restent toujours cohérents par rapport à leur système éthico-esthétique à chaque moment de leur développement/ évolution historique.

\section{Bibliographie}

BAXTIN, M. M. « Formy vremeni i xronotopa v romane. Očerki po istoričeskoj poètike ", Voprosy literatury i èstetiki. Moskva: Xudožestvennaja literatura, 1975.

BAXTIN, M. M. "Otvet na vopros redakcii "Novogo mira" », Èstetika slovesnogo tvorčestva. Moskva: Iskusstvo, 1986.

22 Elisabeth W. Bruss, Autobiographical Acts : the Changing Situation of Literary Genre, Baltimore / London, Johns Hopkins University Press, 1976. 
BAXTIN, M. M. «Problema rečevyx žanrov », Èstetika slovesnogo tvorčestva. Moskva: Iskusstvo, 1986.

BAXTIN M. M. «Problema teksta », Avtor i geroj. K filosofskim osnovam gumanitarnyx nauk. Sankt-Peterburg: Azbuka, «Academia », 2000.

BAXTIN M. M. Problemy poètiki Dostoevskogo, Moskva: Sovetskij pisatel', 1963.

BRUSS, Elisabeth W. Autobiographical Acts : the Changing Situation of Literary Genre. Baltimore / London: Johns Hopkins University Press, 1976.

HOLQUIST, Michael. "Mikhail Bakhtine ", in Histoire de la littérature russe. Le XXe siècle. Gels et dégels. Paris: Fayard, 1990, p. 563-572.

Le dictionnaire historique de la langue française, Alain Rey (éd.). Paris: Dictionnaires le Robert, 2006, t. 2.

SERMAN, Ilya. "Iouri Tynianov ", in Histoire de la littérature russe. Le XXe siècle. La Révolution et les années vingt. Paris: Fayard, 1988, p. 675-688.

TYNJANOV, Ju. N. "O literaturnoj èvoljucii », Literaturnaja èvoljucija. Izbrannye trudy. Moskva: Agraf, 2002.

Recebido em: 29/06/2020

Aceito em: 30/07/2020

Publicado em setembro de 2020 